\title{
Enfermagem e o envelhecer saudável
}

\author{
Nursing Caring for Healthy Aging
}

\section{La enfermería cuidando la salud para un envejecimiento saludable}

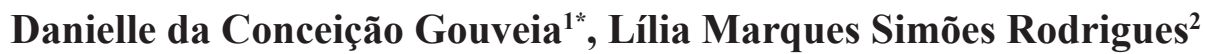 \\ Como citar esse artigo. Gouveia,}

DC; Rodrigues, LMS. Enfermagem e

o envelhecer saudável. Revista PróUniverSUS. 2019 Jan./Jun.; 10 (1): $97-$ 101.

\author{
Resumo
}

O estudo tem como objetivos identificar o papel do enfermeiro no processo do envelhecimento saudável, a sua contribuição para a promoção e a prevenção de saúde da população e analisar as ações de promoção e prevenção à saúde desenvolvida pelos profissionais de enfermagem junto à população. Trata-se de uma pesquisa exploratória e descritiva de natureza qualitativa. Foi utilizado como instrumento de coleta de dados, um questionário semiestruturado, os sujeitos da pesquisa foram oito enfermeiros da Atenção Básica que atuam nas Estratégia de Saúde da Família a pelo menos um ano e que se dispuseram a participar da pesquisa, no município de Vassouras/RJ. Os dados foram analisados pelo método de análise de conteúdo. Com o estudo foi possível reconhecer que a maioria dos profissionais da enfermagem tem o conhecimento do conceito saúde, porém embora tenha relatos da realização de ações para promover a saúde, identificou-se, que há poucas ofertas as ações de promoção implantadas nas unidades.Observando assim, a necessidade de implantação de mais estratégias direcionadas a comunidade que o profissional encontra-se inserida, que fará uma melhor assistência à saúde, contribuindo para a redução de complicações através da educação voltadas a população em um todo, direcionadas para o cuidar da saúde.

Palavras-chave: Enfermagem; Envelhecimento; Saúde .

\begin{abstract}
The objective of this study is to identify the role of nurses in the process of healthy aging, their contribution to the promotion and prevention of health of the population and to analyze the actions of promotion and prevention to health developed by nursing professionals in the population. This is an exploratory and descriptive research of a qualitative nature. A semi-structured questionnaire was used as a data collection instrument, the subjects of the research were eight Primary Care nurses who have been working in the Family Health Strategy for at least one year and who were willing to participate in the study in the municipality of Vassouras / RJ. The data were analyzed by the content analysis method. With the study it was possible to recognize that the majority of nursing professionals have knowledge of the concept of health, but although there are reports of actions to promote health, it was identified that there are few offers the promotion actions implemented in the units. thus, the need to implement more strategies directed to the community that the professional is inserted, which will make a better health care, contributing to the reduction of complications through education directed at the population as a whole, directed towards health care.
\end{abstract}

Keywords: Nursing; Aging; Cheers.

\section{Resumen}

El estudio tiene como objetivos identificar el papel del enfermero en el proceso del envejecimiento saludable, su contribución a la promoción y la prevención de la salud de la población y analizar las acciones de promoción y prevención a la salud desarrollada por los profesionales de enfermería junto a la población. Se trata de una investigación exploratoria y descriptiva de naturaleza cualitativa. Se utilizó como instrumento de recolección de datos, un cuestionario semiestructurado, los sujetos de la investigación fueron ocho enfermeros de la Atención Básica que actúan en las Estrategias de Salud de la Familia a al menos un año y que se dispusieron a participar de la investigación, en el municipio de Vassouras / RJ. Los datos se analizaron mediante el método de análisis de contenido. Con el estudio fue posible reconocer que la mayoría de los profesionales de la enfermería tienen el conocimiento del concepto salud, pero aunque tiene relatos de la realización de acciones para promover la salud, se identificó, que hay pocas ofertas las acciones de promoción implantadas en las unidades. así, la necesidad de implantación de más estrategias dirigidas a la comunidad que el profesional se encuentra inserta, que hará una mejor asistencia a la salud, contribuyendo a la reducción de complicaciones a través de la educación dirigidas a la población en un todo, dirigidas para el cuidado de la salud.

Palabras clave: enfermería; envejecimiento; salud.

Afiliação dos autores:

1 Acadêmico do Curso de Enfermagem, Universidade de Vassouras. RJ, Brasil. Email: danicgouveia@hotmail.com ORCID: https://orcid.org/0000-0002-6709-3171

2 Mestre. Professora e Coordenadora do Curso de Enfermagem, Universidade de Vassouras. RJ, Brasil. Email: liliasrodrigues21@gmail.com ORCID: https://orcid.org/0000-00032979-6316

* Email de correspondencia: danicgouveia@hotmail.com 


\section{Introdução}

A população mundial vem envelhecendo rapidamente em função da diminuição da taxa de fecundidade e do aumento da expectativa de vida. De acordo com dados do Censo Demográfico de 2010, do Instituto Brasileiro de Geografia e Estatística - IBGE, o Brasil, até 2025, será o sexto país do mundo com o maior índice de pessoas idosas. Esta realidade tem determinado uma modificação no perfil demográfico. Segundo ONU, o mundo está em uma transição do processo demográfico, única e irreversível que irá resultar em populações mais velhas em todos os lugares.

À medida que taxas de fertilidade diminuem a proporção de pessoas com 60 anos ou mais deve duplicar entre 2007 e 2050. O Brasil vem envelhecendo de forma rápida e intensa. Nos dias atuais, o país possui a quinta maior população idosa do mundo. ${ }^{1} \mathrm{Com} o$ aumento da expectativa de vida no Brasil, percebese a importância de se conhecer e entender melhor o processo de envelhecimento.

Envelhecimento, do ponto de vista biológico, a perda progressiva das funções. Essa diminuição associada à idade ocorre junto com a redução da fertilidade e o aumento do risco de morte. Oliveira (2017) explica, o envelhecimento pode ser conceituado como um processo dinâmico, no qual as alterações morfológicas, funcionais e bioquímicas ocorrem progressivamente no organismo, tornando-o mais suscetível às agressões intrínsecas e extrínsecas. ${ }^{2}$

Políticas e programas que envolvem a saúde do idoso foram criados para garantir os direitos dos idosos na sociedade.

Segundo a legislação brasileira, idosa é a pessoa com 60 anos de idade ou mais, conforme dispõe a Política Nacional do Idoso (PNI), Lei n. 8.842 de 4 de novembro de 1994, regulamentada pelo Decreto $n$. 1.948 de 20 de dezembro de $1996 .^{3}$

A Política Nacional do Idoso (PNI), Lei $8.842 / 1994$, criou normas para os direitos sociais dos idosos, garantindo autonomia, integração e participação efetiva como instrumento de cidadania. Seu objetivo foi criar condições para promover longevidade com qualidade de vida, com ações não apenas para os idosos, mas para aqueles que também irão envelhecer. ${ }^{3}$

Já o Estatuto do Idoso (Lei no 10.741/2003) veio também reforçar e garantir os direitos fundamentais dos idosos, mas estabelece ordem de execução e punição quando desrespeitados estes direitos. ${ }^{4}$

A Organização Mundial da Saúde (OMS) 1948, define: "A saúde é um estado de completo bem-estar físico, mental e social e não apenas a ausência de doenças e enfermidades". E também defendida pela Constituição federal de 1988, a saúde é direito de todos e dever do Estado. Para garantir esse direito, foi criado o Sistema
Único de Saúde (SUS), que se baseia: universalidade, igualdade de acesso e integralidade no atendimento. ${ }^{5}$

$\mathrm{O}$ processo de envelhecimento acontece de maneira gradual, ativa e irreversível, com uma série de mudanças genético-biológicas, psicossociais e fisiopatológicas, Com isso, muitas doenças podem surgir e gerar limitações ao idoso. É nesse contexto que os profissionais da saúde estão inseridos, a fim de promover a saúde do idoso e fazer com que o envelhecimento seja saudável e ativo como preconizado nas políticas públicas de saúde. A promoção da saúde visa a diminuição da vulnerabilidade e dos riscos à saúde da população por meio da participação e controle social. ${ }^{6}$

O envelhecimento populacional traz consigo problemas de saúde que desafiam os sistemas de saúde. Envelhecer não significa necessariamente adoecer. A menos que exista doença associada, o envelhecimento está associado a um bom nível de saúde. Além disso, os avanços no campo da saúde e da tecnologia permitiram para a população com acesso a serviços públicos ou privados adequados, uma melhor qualidade de vida nessa fase. Com isso, é fundamental investir em ações de prevenção ao longo de todo o curso de vida, em virtude do seu potencial para "resolver os desafios de hoje e, de forma crescente, os de amanhã".

Para o sistema de saúde, esse impacto do envelhecimento populacional traz importantes mudanças que necessitam dos serviços da saúde, como o aumento dos riscos das doenças crônicodegenerativas, ${ }^{8}$ à medida que os indivíduos envelhecem pode ocorrer o aparecimento das Doenças e Agravos Não Transmissíveis (DANT), sendo as mais comuns em idosos: as principais causas de morbidez, deficiências e mortalidade entre os adultos e idosos. ${ }^{9}$

Portanto, o aumento da incidência dessas doenças na população que está envelhecendo poderá provocar enormes custos humanos e sociais para o controle e tratamento dessas doenças, necessitando de uma quantidade desproporcional de recursos financeiros e humanos que poderiam ser destinados a outros problemas de saúde de outras faixas etárias, assim como investir em medidas de educação, promoção e prevenção em saúde na atenção primária à saúde. ${ }^{10}$

O estudo têm como objetivos identificar o papel do enfermeiro no processo do envelhecimento saudável, a sua contribuição para a promoção e a prevenção de saúde da população e analisar as ações de promoção e prevenção à saúde desenvolvida pelos profissionais de enfermagem junto à população.

\section{Metodologia}

Trata-se de uma pesquisa exploratória e descritiva, de cunho qualitativo, cujo objetivo principal é descrever como os profissionais de enfermagem atuam 
para o processo do envelhecimento saudável.

Dos 24 (vinte e quatro) enfermeiros (as) que atuam nas ESF quanto na UBS do município da Região Sul Fluminense do estado Rio de Janeiro, 8 enfermeiros responderam às perguntas, 3 atuavam a menos de um ano naquela unidade, 3 estavam de licença médica e 3 de férias e 7 não aceitaram participar da pesquisa. Como critérios de inclusão, ficou estabelecido: ser enfermeiro (a) e estar atuando na atenção básica há pelo menos um ano. Foram excluídos enfermeiros que estavam atuando menos 01 (um), os que estiverem de férias, os que encontrarem de férias e que não concordaram com a pesquisa. Os sujeitos da pesquisa são profissionais que atuam na atenção básica, onde sua prática de trabalho deve ser baseada na prevenção e promoção da saúde, tornando assim capazes de fornecer informações relevantes que respondam aos objetivos da pesquisa.

A coleta dos dados foi realizada no período compreendido entre os meses de agosto e setembro de 2018. Foi utilizado como instrumento de coleta de dados, um questionário semiestruturado, sendo esse dividido em duas etapas: identificação com os seguintes itens idade; sexo; tempo de: formado, atuação profissional e atuação na estratégia de saúde da família onde atua no momento da pesquisa, a segunda etapa foi composta de perguntas relacionadas ao estudo, como: Qual a definição de saúde?, O que é o envelhecer saudável?, $\mathrm{O}$ que os profissionais entendem sobre os programas e políticas sociais voltados para a promoção de saúde?, Quais as ações educativas que a unidade oferece para a população? Quais as estratégias utilizadas pelos enfermeiros para promover o envelhecimento saudável? Sendo entregue aos enfermeiros nas unidades de saúde da família e respondido na presença das pesquisadoras, evitando possíveis interferências externas.

Para preservar o anonimato, os participantes enfermeiros foram identificados com a letra $E$ (Enfermeiro), seguido de um número de identificação de I a VIII.

O projeto foi submetido à avaliação pelo Comitê de Ética em Pesquisa da Universidade de Vassouras. Aprovado com o número do parecer: 2.784.505. Atendendo as resoluções éticas e exigências da Resolução 466/2012, do Conselho Nacional de Saúde.

Aplicou-se a técnica de Análise de Conteúdo, havendo a releitura do material para a categorização das respostas dadas a partir de números, que segundo Bardin (2009) pressupõe algumas etapas, como: pré-analise, exploração do material ou codificação, tratamento dos resultados.

\section{Resultados e Discussão}

A pesquisa foi realizada com os enfermeiros da Atenção Básica do município de Vassouras que atuavam nas Unidades de Estratégia da Família a pelo menos um ano, que não estavam de férias e nem de licença médica e que se dispuseram a participar do estudo. Sendo o total de sujeitos que participaram da pesquisa 8 enfermeiros que corresponde a $100 \%$ dos participantes.

Dos entrevistados, $100 \%$ eram do sexo feminino. Lopes e Leal (2005) relatam que em relação aos contingentes do gênero feminino na profissão, pesquisas que vem sendo realizado ao longo dos anos, e vem constatando que o predomínio do sexo feminino no exercício da profissão ainda é marcante, em todas as categorias de trabalhadores de enfermagem.

Apresentaram como características uma média de idade de $37,5 \%$ de 25 a 30 anos, $12,5 \%$ entre 31 a 35 anos, 50\% entre 36 a 40 anos. Quanto aos anos de formação como enfermeiro, evidenciou-se que 12,5\% possui até 5 anos, 50\% entre 6 e 10 anos, 25\% entre 11 e 15 anos, 12,5\% acima de 16 anos. Constatando-se que a maioria possui entre 6 a 10 anos de formados. Em relação ao tempo de atuação $62 \%$ atua até 5 anos, 25\% de 6 a 14 anos e $12,5 \%$ mais de 15 anos. Em relação ao tempo que atua na unidade que está inserido, $37,5 \%$ atua entre 1 a 2 anos, 37,5\% entre 3 a 4 anos, 37,5 acima de 5 anos. A literatura aponta que a rotatividade dos profissionais compromete o seu vínculo com a comunidade $\mathrm{e}$ a qualidade da assistência. E também, mudanças constantes dos profissionais na equipe acarretam sobrecarga de trabalho para os que permanecem e exige o treinamento de novos profissionais, aumentando os custos e fragilizando os processos de trabalho. ${ }^{11}$

Sobre os que possuem pós graduação, das entrevistadas, $75 \%$ responderam que sim, e $25 \%$ não. De acordo com a literatura a qualificação tem contribuído para o aperfeiçoamento e mudanças na prática profissional, associando um melhor resultado do serviço, com a melhor qualificação de seus profissionais. ${ }^{12}$ Ainda, a qualidade da formação do profissional pode fortalecer uma atuação baseada na concepção de modelo assistencial, organização do serviço ou prática de trabalho. ${ }^{11}$

Os que responderam sim citaram como especializações realizadas: Enfermagem ginecológica e obstétrica; Gestão de redes de atenção à saúde; Gestão em saúde Pública, Especialização em saúde da família, UTI neonatal e ginecologia e obstetrícia.

A partir dos dados coletados desta pesquisa foi possível reconhecer como os enfermeiros da atenção básica de saúde, no município de Vassouras, vem lidando com o conceito saúde e perceber como eles vem contribuindo e quais ações vem sendo desenvolvidas nas unidades para a promoção e prevenção de saúde da população.

O material submetido a análise de conteúdo originou a seguinte categoria: Programas e políticas voltados à promoção de saúde.

A criação de políticas e programas de saúde, 
surgiram com um dos objetivos, de melhorar à saúde da população que vem envelhecendo. A atenção à saúde em políticas de promoção, proteção e recuperação da saúde.

A Política Nacional de atenção básica é formada por um conjunto de ações de saúde que abrange a promoção e a proteção da saúde, a prevenção de agravos, o diagnóstico, o tratamento, a reabilitação, a redução de danos e a manutenção da saúde com o objetivo de desenvolver uma atenção integral que impacte na situação de saúde e autonomia das pessoas e nos determinantes e condicionantes de saúde das coletividades. ${ }^{13}$

O Programa Nacional de Melhoria do acesso e da qualidade da Atenção Básica (PMAQ), é um exemplo de programa que envolve toda a equipe de saúde da atenção básica, através de incentivo financeiro aos gestores que foi criada com o intuito de melhorar a qualidade to atendimento da população, através de estratégias, acompanhamento e avaliação das atividades realizadas pelos profissionais da saúde. ${ }^{13}$

E uma das principais formas de colocar em prática essas ações impostas por políticas e programas, são as através da atenção básica, desenvolvendo ações de promoção da saúde e prevenção de doenças. ${ }^{14}$

Buscando por esse ponto, foi perguntado no questionário para as enfermeiras a sua opinião a respeito desse tema. Na visão dos profissionais os programas e políticas de saúde são essenciais, porém, ainda não funcionam de forma adequada.

Entendemos que são fundamentais para a promoção da saúde e uma oferta de uma assistência qualificada. EI São programas excelentes desde que saiam do papel e que dê estrutura para trabalhar. EII Entendo como uma melhoria na qualidade de vida do usuário, que através de práticas educativas, garantem empoderamento sobre sua saúde bem como, a melhoria dela. EIII Os programas ainda são falhos e com pouca aceitação da população. Ainda precisam melhorar os programas. EVIII

A partir da criação do SUS, ao longo dos anos foram implementadas diversas políticas, programas, ações e práticas, priorizando a promoção de saúde e prevenção de doenças. Portanto, ainda é constante a assistência focada mais às doenças.

Um dos motivos do não funcionamento adequado de práticas de promoção da saúde acredita, é devido baixa precisão na definição do que seja, de fato, promoção da saúde; pouca clareza do que se espera que as equipes de atenção primária desenvolvam quanto à promoção da saúde; e por ser precárias as iniciativas de avaliação sobre o que se pensa e o que se faz em Atenção Primária à Saúde no que se refere à promoção da saúde..$^{15}$

Portanto, apesar da criação de políticas e programas, os profissionais da enfermagem precisam incorporar um olhar qualificado e direcionado, que fará a assistência à saúde mais eficiente e eficaz, contribuindo para a redução de complicações e aparecimento de agravos na população com ênfase na promoção de atividade física, na promoção de hábitos saudáveis de alimentação e vida, controle do tabagismo; controle do uso abusivo de bebida alcoólica; e cuidados especiais voltados ao processo de envelhecimento.

Cabe aos profissionais desenvolverem ações de promoção da saúde, incentivando hábitos de vida saudáveis, como alimentação adequada, prática de atividade física e práticas corporais.

A oferta de ações de saúde está focada em indivíduos com problemas crônicos e mudanças de hábitos de vida inadequados, e não na promoção da saúde em população saudável. Estudos realizados no Brasil verificaram que indivíduos adultos com doenças crônicas (hipertensão e diabetes) e com excesso de peso receberam mais orientações sobre alimentação saudável e prática de atividade física do que os indivíduos sem doenças crônicas ou excesso de peso. ${ }^{16}$

\section{Conclusão}

O presente estudo, pode destacar a percepção dos enfermeiros entrevistados, sobre o conceito saúde, demostrando assim que o assunto deve ser mais explorado em estudos futuros. Porém, há necessidade de aprimorardo aumento de ações direcionadas para o cuidar da saúde. Em relação às estratégias de promoção de saúde desenvolvidas pelos enfermeiros na atenção básica, identificou-se, que há poucas ofertas.

Para que essas estratégias possam ser desenvolvidas, foram criadas ferramentas que são as políticas e programas com o objetivo de melhorar a qualidade de vida da população. Porém, somente os instrumentos,não são suficientes, é imprescendível a formação adequada e o conhecimento dos profissionais para atuarem no campo da educação em saúde com o enfoque não somente no controle e tratamento de morbidades, mas em ações que promovem a saúde. Para isso, é preciso que sejam realizadas um planejamento de ações a serem implantadas a comunidade que o profissional encontra-se inserido, um monitoramento dessas ações e a avaliação das estratégias implantadas junto a comunidade.

Dentre as ações que podem ser desenvolvidas estão: as que atendam as necessidades da população e voltadas à transformação dos comportamentos dos indivíduos, focando na mudança dos estilos de vida como, hábitos de vida saudáveis, estímulos a práticas nutricionais; prática corporal/atividade física; prevenção e controle do tabagismo; ações específicas de detecção precoce de hipertensão e diabetes, atividades como 
avaliação antropométrica, redução da morbimortalidade em decorrência do uso abusivo de álcool e outras drogas, redução da morbimortalidade por acidentes de trânsito, prevenção da violência e estímulo à cultura de paz e promoção do desenvolvimento sustentável. E questões relacionadas com a qualidade de vida como: saneamento, boas condições de trabalho e moradia, educação, apoio social às famílias, entre outros.Podendo essas atividades serem realizadas nas escolas e comunidades em que os indivíduos se situam, levando aconselhamento a comunidade em relação aos benefícios que podem apresentar essas ações para uma melhor qualidade de vida. Desenvolver palestras, grupos de apoio, onde os profissionais possam interagir com a comunidade de forma a motivar a participação e integração dos inndivíduos e desenvolver conhecimento de maneira rápida e de fácil entendimento, para que assim haja conscientização da população sobre a importância da realização do auto cuidado, mostrando que é mais fácil e de menor custo o cuidar da saúde.

Espera-se com este estudo contribuir para ampliar a compreensão dos profissionais sobre a importância do cuidado aos indivíduos saudáveis e instrumentalizar os enfermeiros das Estratégias de Saúde da Família a atuar de forma a atender a sociedade com o enfoque não somente no controle e tratamento de morbidades, mas também em educação voltadas a população em um todo direcionadas para a promoção da saúde,proporcionando assim, uma assistênciaà saúde de qualidade, contribuindo para a redução de complicações e proporcionando a conquista da longevidade com qualidadeaos usuários

\section{Referências}

1. Lima, Cristiane do Carmo Silva. Lei n ${ }^{\circ} 13.466 / 2017$ e a alteração do estatuto do idoso: uma contradição ao rol de prioridade especial para pessoas com mais de 80 anos. Revista Cadernos Ibero-Americanos de Direito Sanitário. 2018 jan./mar, 7(1): 147.

2. Sharon, L. Lewis; et al.Tratado de enfermagem médicocirúrgica:avaliação e assistência dos problemas clínicos.8ed, vol1. Rio de Janeiro: Elsevier, 2013.

3. Brasil. Constiuição da República Federativa do Brasil. 5 de outubro de 1988. Brasília: Senado Federal, 2015.

4. Brasil. Ministério da saúde. Diretrizes para o cuidado das pessoas idosas no SUS: proposta de modelo de atenção integral xxx congresso nacional de secretarias minicipais de saúde. Brasília, 2014

5. OMS. Organização Mundial da Saúde. Constituição da Organização Mundial da Saúde (OMS/WHO). 1948.

6. Mallman, Danielli Gavião et al. Educação em saúde como principal alternativa para promover a saúde do idoso. Ciência \& Saúde Coletiva, 2015; 20:1763-1772.

7. Miranda, Gabriella Morais Duarte; Mendes, Antonio da Cruz Gouveia; Da Silva, Ana Lucia Andrade. O envelhecimento populacional brasileiro: desafios e consequências sociais atuais e futuras. Revista Brasileira de Geriatria e Gerontologia,2016;19(3):507-19.

8. Nunes, Vilani Medeiros de Araújo. Avaliação gerontológica multidimensional das condições de saúde de idosos residentes em instituições de longa permanência. 2012.

9. Medeiros, W. M. D. C. Acidentes de transporte terrestre, não fatais, no Brasil: fatores associados e efeitos sobre a percepção do estado de saúde das vítimas. 2017

10. Mendes Tozim, B., Giglio Furlanetto, M., Moraes de Lorenzo França, D., Morcelli, M. H., \& Tavella Navega, M. Efeito do método Pilates na flexibilidade, qualidade de vida e nível de dor em idosos. ConScientiae Saúde. 2014; 13(4).

11. Scabelo Galavote, H., Zandonade, E., Pinheiro Garcia, A. C., de Souza Silva Freitas, P., Seidl, H., Caran Contarato, P., \& de Cássia Duarte Lima, R. O trabalho do enfermeiro na atenção primária à saúde. Escola Anna Nery Revista de Enfermagem, 2016; 20(1):90-8.

12. Oliveira, Mariana Policena Rosa de et al. Training and qualification of health professionals: Factors associated to the quality of primary care. Revista Brasileira de Educação Médica, 2016; 40(4):547-59.

13. Brasil. Ministério da Saúde. Política Nacional de Atenção Básica. Programa Nacional de Melhoria do acesso e da qualidade da Atenção Básica (PMAQ). Brasília, DF, 2012.

14. Häfele, V., \& Siqueira, F. Aconselhamento para atividade física e mudança de comportamento em Unidades Básicas de Saúde. Revista Brasileira de Atividade Física \& Saúde; 2016;21(6):581-92.

15. Medina, M. G., Aquino, R., Vilasbôas, A. L. Q., Mota, E., Júnior, P., Pereira, E., ... \& Pinto, I. C. D. M. Promoção da saúde e prevenção de doenças crônicas: o que fazem as equipes de Saúde da Família? Saúde em Debate, 2014; 38:69-82.

16. Kessler, M., Lima, S. B. S. D., Weiller, T. H., Lopes, L. F. D., Ferraz, L., \& Thumé, E. Longitudinality in Primary Health Care: a comparison between care models. Revista Brasileira de Enfermagem, 2018;71(3):106371 . 